\title{
Massive star formation through accretion and binary mergers
}

\author{
Ian A. Bonnell \\ School of Physics and Astronomy, University of St Andrews, North Haugh, St Andrews, Ky16 \\ 9SS, UK \\ email: iab1@st-and.ac.uk
}

\begin{abstract}
I review the formation of massive stars in the context of a forming stellar cluster. High-mass stars form in the centre of stellar clusters and thus must be understood in the context of low-mass star formation. Furthermore, they are predominantly in binary systems making further constraints on the formation mechanism. The fragmentation of a turbulent molecular cloud produces a large number of stars with initial masses close to the Jeans mass of the cloud. These stars fall together to form small-N clusters that grow through the infall of gas and stars into the cluster's potential well. Competitive accretion in clusters produces high-mass stars in the cluster centre and a full initial mass function of lower-mass stars. Massive star formation is a process that occurs in the cores of stellar clusters and commonly produces close binary systems. Accretion also forces the cluster to contract, increasing the stellar densities to the point where stellar collisions may occur. Furthermore, accretion in clusters reproduces the high binary frequency of massive stars. Systems evolve from low-mass wide binaries to high-mass close binaries due to gas accretion. This evolution can produce very tight binaries that are expected to merge to form the most massive stars. Binary mergers require stellar densities of order $10^{6}$ stars $\mathrm{pc}^{-3}, 100$ times smaller than is required for single-star collisions.
\end{abstract}

\section{Motivation}

Massive star formation is a process that occurs in the cores of stellar clusters (Clarke et al. 2000; Lada \& Lada 2003). Massive stars are almost never formed in isolation (cf. De Wit this proceedings) but predominantly form in large stellar clusters that have plethora of low-mass stars to comprise a full initial mass function (IMF). In fact, Testi et al. (1998) have shown that there appears to be a correlation with the stellar density or richness of a system with the mass of the most massive object that it contains. This suggests a physical requirement for the formation of massive stars. It is therefore essential to study massive star formation in the context of low-mass star formation and a physical process that produces a full IMF. A further complication to any model of massive star formation is that most massive stars are in binary systems (Mason et al. 1998; Preibish et al. 1999; Garcia \& Mermilliod 2001). These systems are often very close, with separations $\lesssim 1 \mathrm{AU}$, and companions that are also high-mass stars (Garmany, Conti Massey 1980; Bonanos \& Stanek 2005).

\subsection{Problem I: Forming Massive Stars}

The formation of high-mass stars is a large unknown in modern astronomy. While our understanding of the formation of low-mass stars has improved dramatically over the past decade (Larson 2003), we still do not know whether massive star formation is basically a scaled-up version of low-mass star formation or if it results from a dramatically different process (Stahler, Palla \& Ho 2000; Bally \& Zinnecker 2005). The basic questions concern 
how mass is added and how it interacts with the large radiation pressure from the high stellar luminosity once the star reaches masses in excess of $10 M_{\odot}$. Models of scaled-up low-mass star formation have repeatedly shown that if the gas contains typical interstellar dust, then the radiation pressure deposits sufficient momentum into the dust (well coupled to the gas) to repel the infalling gas (Yorke \& Krügel 1977; Wolfire \& Casinelli 1987; Beech \& Mitalas 1994; Edgar \& Clarke 2004).

Potential solutions to this problem include accretion through a disc (Yorke \& Sonnhalter 2002), accretion overpowering the radiation pressure (McKee \& Tan 2003), and massive star formation through mergers of (dust free) stars (Bonnell, Bate \& Zinnecker 1998; Bonnell \& Bate 2002). Disc accretion can help in two ways. Firstly, the matter accretes through a small solid angle and is thus less exposed to the radiation pressure. Secondly, as young stars are generally thought to be rapidly rotating, the star can be significantly cooler at the equator than at its poles. Thus, there is less radiation pressure in the equatorial regions to oppose the accretion. Using this last factor in their models as well as a sophisticated treatment of the radiation transfer, Yorke \& Sonnhalter (2002) showed that it is possible to accrete up to masses of the order $30 \mathrm{M}_{\odot}$ before the radiation pressure repels the infalling envelope. McKee \& Tan (2003) have speculated that a very concentrated centrally condensed core will provide large accretion rates onto a forming massive star which will then overpower the radiation pressure of the growing star. There are two limitations to this process. Firstly, such a core, even if centrally condensed and supported by turbulence, is very likely to fragment and form a small stellar cluster (Dobbs, Bonnell \& Clark 2005). Secondly, the implied accretion rates are similar to the models of Yorke \& Sonnhalter (2002) which show that the infalling envelope is indeed repelled once the star attains masses of order $30 \mathrm{M}_{\odot}$, although this may be overcome by Raleigh-Taylor instabilities in the accretion flow (Krumholz this volume). Of more difficulty is how the required physical conditions can arise in the dense cores of stellar clusters without being disrupted by the tidal forces from the other stars.

The third potential solution involves the merger of lower-mass stars in a dense stellar cluster (Bonnell et al. 1998). While certainly the most exotic of the three, it does have the attraction that any dust will be destroyed in the lower-mass stars and thus there is no problem with the radiation pressure from the forming massive star. The obvious difficulties with this model is that it requires very high stellar densities (of order $10^{8}$ stars $\mathrm{pc}^{-3}$ ) in order for stellar collisions to be sufficiently common to form massive stars in less than $10^{6}$ years. Although such densities are $10^{3}$ times higher than generally found in the cores of young stellar clusters, they are not inconceivable as the dynamics of accretion can induce the core of a cluster to contract significantly (Bonnell et al. 1998; Bonnell \& Bate 2002).

\subsection{Formation of Close Binary Stars}

Forming close binary stars systems is difficult even amongst lower-mass stars, The reason for this is that if the components form through fragmentation (eg, Boss 1986; Bonnell 1999), then the Jeans radius at the point of fragmentation must be smaller than their separation,

$$
R_{\text {sep }} \gtrsim 2 R_{J} \propto T^{1 / 2} \rho^{-1 / 2} .
$$

As above, this implies a high gas density and thus a low Jeans mass,

$$
M_{*} \approx M_{J} \propto T^{3 / 2} \rho^{-1 / 2}
$$


This results in the mass of the stars being directly related to their separation,

$$
R_{\mathrm{sep}} \propto \frac{M_{*}}{T},
$$

such that close systems have very low masses. For example, if the typical 30 AU binary has solar mass components, then a $1 / 3$ AU binary should have components of $0.01 \mathrm{M}_{\odot}$. Forming close binary stars in situ is therefore difficult as it requires subsequent accretion to reach stellar masses (Bonnell \& Bate 1994). An alternative is that the components form at greater separation and then are brought together. Recent simulations of star formation in a cluster environment have shown that close binaries can result from the induced evolution of wider systems (Bate, Bonnell \& Bromm 2003a). The binaries evolve due to gas accretion and dynamical interactions with other stars. Can the same processes explain high-mass close binary systems?

\section{Accretion and Binary Evolution}

Accretion onto binary systems has the potential of forming close systems out of wider systems at the same time as forming higher-mass components. In order to see this, let us consider the angular momentum of a binary system,

$$
L \propto M_{\mathrm{bin}}^{3 / 2} R_{\mathrm{bin}}^{1 / 2} .
$$

If the accreted material has zero net angular momentum, as is expected if it infalls spherically symmetric, then $L \approx$ constant, and the binary separation should be a strong function of the mass,

$$
R_{\mathrm{bin}} \propto M_{\mathrm{bin}}^{-3} .
$$

In a turbulent medium, as expected here, the angular momentum of each parcel of infalling gas is essentially randomly oriented. The net angular momentum will then do a random walk with increasing mass such that

$$
L \propto M_{\mathrm{bin}}^{1 / 2},
$$

and thus

$$
R_{\text {bin }} \propto M_{\text {bin }}^{-2}
$$

Under these basic assumptions, we can see that accretion onto a binary system can significantly decrease its separation at the same time as it increases its mass (Bate \& Bonnell 1997; Bate 2000).

\section{Accretion and the Evolution of Stellar Clusters}

The evolution of a stellar cluster under the effects of accretion is analogous to that of the simple binary case above. A cluster of stars that accretes gas will become more bound and therefore have to contract to reestablish virial equilibrium (Bonnell et al. 1998). Depending on the net momentum gained with the mass accreted, the cluster can contract as dramatically as in the binary case. The most extreme case, $R \propto M^{-3}$ results in an increase in the stellar density of $n \propto M^{9}$ and thus even moderate densities can easily evolve to extreme ones in which stellar collisions can occur. Simulations of this process do indeed show an increase in the stellar density of order $10^{5}$ while the mass is increased by only a factor of a few (Bonnell \& Bate 2002). Even the more moderate $R \propto M^{-2}$ case as expected for a turbulently supported cloud will induce an increase in the stellar density of $n \propto M^{6}$. In this case, an increase in average stellar mass by a 


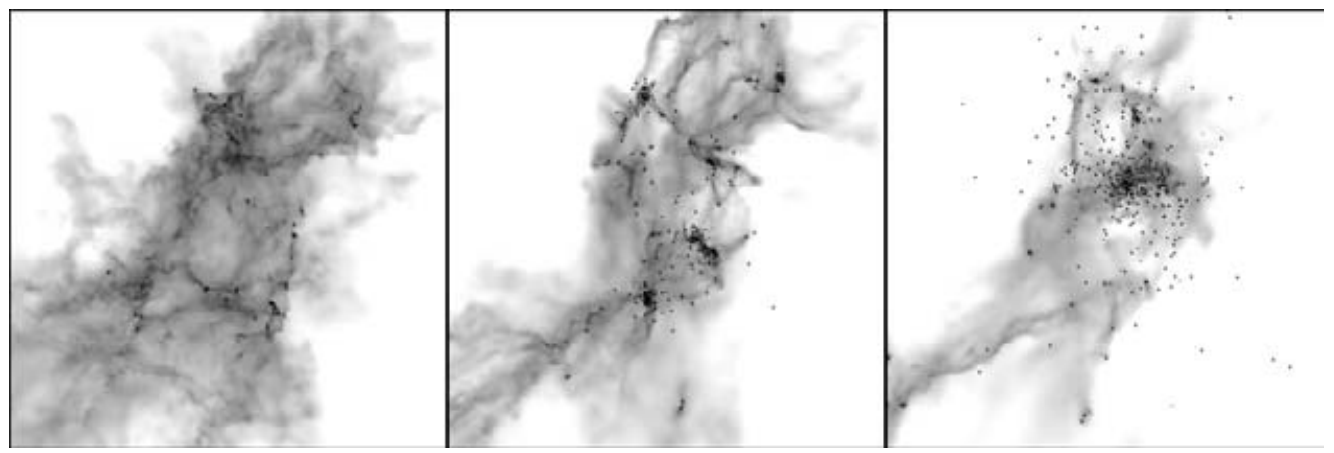

Figure 1. The fragmentation of a $1000 \mathrm{M}_{\odot}$ molecular cloud and the formation of a stellar cluster containing 419 stars (Bonnell et al. 2003).

factor of $\approx 3$ increases the stellar density by $\approx 10^{3}$ while an increase of the mass by 5 (as appropriate for the core of the ONC) increases the stellar density by $>10^{4}$.

\section{The Formation of Stellar Clusters}

The fragmentation of a molecular cloud to form a stellar cluster has been the subject of several studies (Boss 1996; Klessen et al. 1998; Klessen \& Burkert 2000; Bate, Bonnell \& Bromm 2003b; Bonnell, Bate \& Vine 2003). In recent work, the fragmentation is due to turbulently generated structure in molecular clouds. For example, in Bonnell et al. (2003), the fragmentation of a $1000 \mathrm{M}_{\odot}$ cloud occurs as the turbulence generates filamentary structure (see Figure 1). The filaments fragment to form individual stars which fall into local potential wells forming small clusters. These clusters grow by accreting further stars and gas and eventually merge to form a large stellar cluster containing some $>400$ stars. The stars all form with masses typical or less than the initial Jeans mass of the cloud which was chosen to be $1 M_{\odot}$. Subsequent gas accretion forms the higher-mass stars. This simulation is also noteworthy as it was the first that was sufficiently large to populate a full IMF from low-mass to high-mass stars.

Competitive accretion in stellar clusters occurs as individual stars compete gravitationally for the reservoir of gas. As fragmentation is highly inefficient (eg Bate et al. 2003b; Bonnell et al. 2003), there remains a large mass of gas that can dominate the potential. Accretion of this gas can then determine the final stellar masses. Numerical studies have shown that competitive accretion results in a large range of masses, with stars in the centre of the cluster, the deepest part of the potential, accreting more gas due to their location (Bonnell et al. 1997; Bonnell et al. 2001a). Furthermore, competitive accretion can explain the initial mass function as it predicts a two power-law IMF. Low-mass stars accrete the majority of their mass in a gas dominated regime where tidal forces limit their eventual mass, resulting in a shallow IMF (Bonnell et al. 2001b). Higher-mass stars accrete their mass in the stellar dominated cores of the clusters with accretion rates determined by a Bondi-Hoyle-type process, resulting in a steeper IMF. It is worthwhile noting that the accretion rates are determined by the infall rate into the clusters. The gas cannot stagnate inside the cluster but instead flows into the potential well of the cluster and then into the local potential of an individual star.

Another outcome of competitive accretion in stellar clusters is that it increases the stellar density. This occurs as the mass loading increases the binding energy of the cluster. Even if significant momentum is also accreted, the effect is to force the cluster to contract and reviralise. This increases the stellar density. In the simulation of Bonnell, Bate \& 

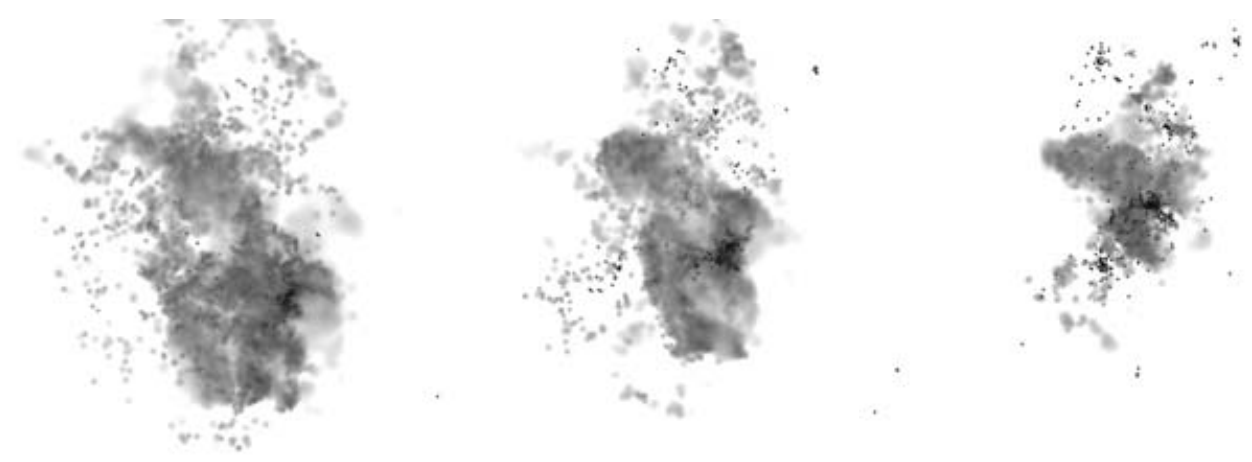

Figure 2. The mass that forms the most massive $\left(30 \mathrm{M}_{\odot}\right)$ star is plotted at three different times during the formation of a stellar cluster. Note that the gas is very distributed when star formation is initiated (left) and when the cluster is growing through the infall of newly formed stars and gas (middle and right). The vast majority of the final mass is due to competitive accretion in a clustered environment (Bonnell et al. 2004).

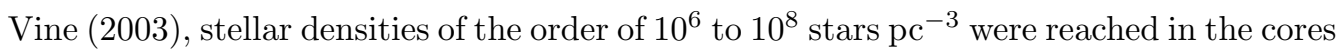
of the clusters. In fact, even these high densities are lower-limits due to the gravitational softening used (at $r \leqslant 160 A U$ ).

\subsection{Accretion and the Formation of Massive Stars}

The numerical simulations discussed above provides a framework in which to understand the formation of massive stars. In this scenario, the massive stars form due to competitive accretion onto the core of the cluster in which the massive star is forming. The Lagrangian nature of the SPH hydro code used for the simulations allows for the decomposition of the mass accretion history of each star. In this way we have been able to analyse where the mass of the massive stars comes from and thus the potential for accretion to form massive stars, and close binaries amongst the massive stars.

We found that the vast majority of the mass which comprises the massive stars comes from large distances and is accreted onto the star after a stellar cluster has formed (Figure 2, Bonnell, Vine \& Bate 2004) . The initial fragment mass which forms the star is of low mass, typical to the mean stellar mass. The infalling gas then has to pass through the cluster to be accreted by the central massive star. We also found that the infalling gas is accompanied by newly formed stars such that the formation of a massive star is a necessary byproduct of the formation of a stellar cluster. It should be noted that these simulations neglect the effect of radiation pressure from the massive stars, or equivalently assume that accretion through a disc (Yorke \& Sonnhalter 2002) occurs.

We have seen above that the formation of massive stars is due to the infall of gas into a stellar cluster and the subsequent competitive accretion inside the cluster. in addition to gas, newly formed stars also infall into the cluster, increasing the number of stars, and hence the stellar densities. Investigating the evolution of the individual clusters, we find a correlation in the richness of the cluster and the mass of the most massive star therein (Figure 3). This can be understood in the following way. Given an effective initial efficiency of fragmentation, for every star that falls into the cluster a certain amount of gas also enters the cluster. This gas joins the common reservoir from which the most massive star takes the largest share in this competitive environment. Thus, the mass of the most massive star increases as the cluster grows in numbers of stars. We therefore have a prediction from this model that there should exist a strong correlation between 


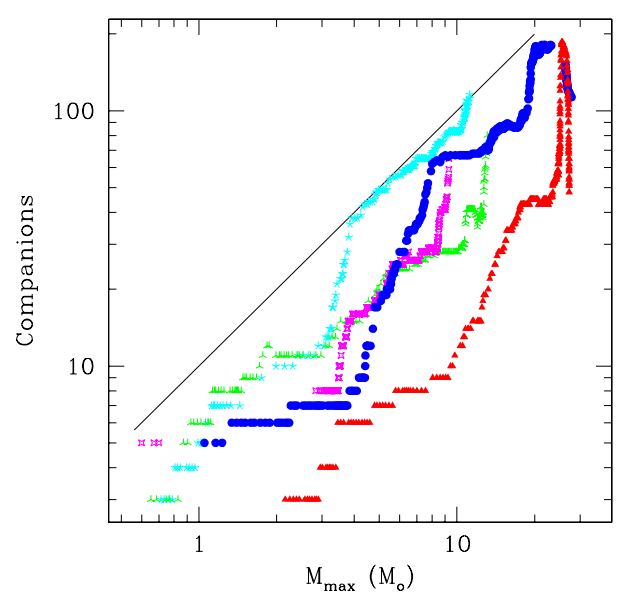

Figure 3. The number of stars in a cluster is plotted against the mass of the most massive star therein showing a direct link between the formation of massive stars and the formation of stellar clusters (Bonnell et al. 2004).

the mass of the most massive star and the number of stars (or the total mass) in the cluster (Figure 3, Bonnell, Vine \& Bate 2004).

\section{Binary Formation and Evolution}

The numerical simulations detailed above form a significant number of binary systems amongst the massive stars. The binary systems generally form through three-body capture in the cores of the clusters. During the earlier stages of the cluster formation, the small number of stars contained in each subcluster allows for significant interactions and a relatively low velocity dispersion such that three-body capture is common and few of the eventual higher-mass stars are not in binary or multiple systems.

Once the binary has formed, continuing accretion onto it increases the masses of the individual stars. Of equal importance is the effect of the accretion on the binary's separation. As the infalling gas has no correlation with the binary, its specific angular momentum is uncorrelated with that of the binary's. Thus, accretion does not significantly increase the binary's angular momentum and the separation of the binary decreases as mass is added (see also Bate 2000). This process is illustrated in Figure 4 which shows the evolution of the binary mass versus orbital separation for one of the high-mass binary systems formed in the simulation (final masses 17 and $10 \mathrm{M}_{\odot}$ ). The system originates as a low-mass wide binary and evolves towards higher masses and smaller separations. Once the separations are $\leqslant 160 \mathrm{AU}$, the gravitational smoothing stops the binary from evolving to smaller separations.

The early stages of the evolution are well parameterised by $R_{\text {bin }} \propto M_{\text {bin }}^{-2}$ as expected for accretion from a turbulent medium where each gas parcel has randomly oriented angular momentum (see $\S 2$ above). Unfortunately, the binary's orbital evolution quickly enters the regime where the gravitational forces are smoothed (160 AU). In spite of this, we can still hope to extract information as to what the true binary separations should be. This is possible as the orbital parameters depend solely on the system's mass, angular momentum and total energy, which are all directly calculable from the simulation (Bonnell \& Bate 

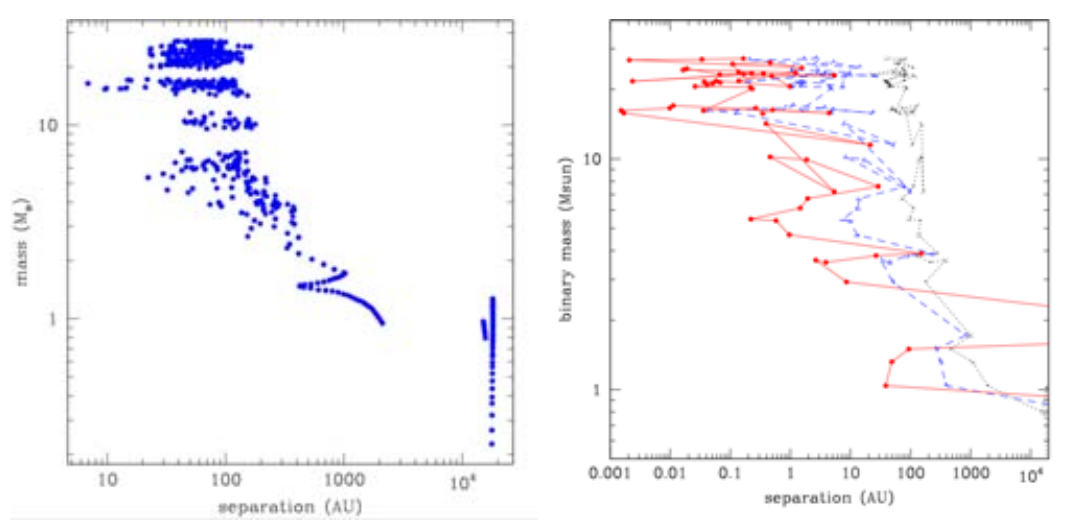

Figure 4. The evolution of a massive binary's mass versus its separation due to the combined effect of accretion and stellar interactions. The gravitational softening is set at $180 \mathrm{AU}$, such that all separations below this value are upper limits. The panel on the right shows the evolution of the same system's semi-major axis and periastron separation as deduced by the specific angular momentum and energy of the system (Bonnell \& Bate 2005). We see that the separation decreases for the more massive systems which have undergone the most accretion.

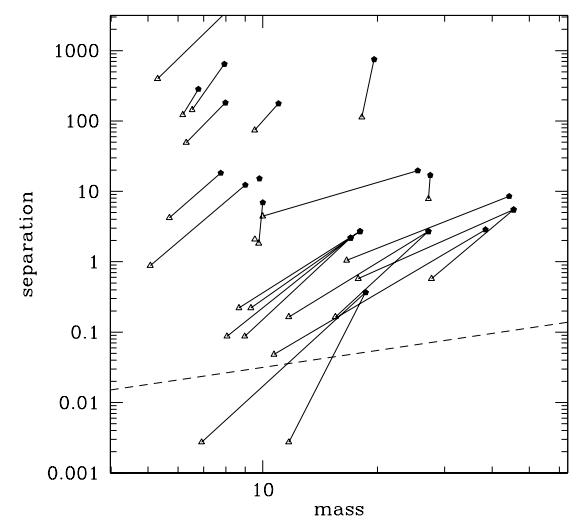

Figure 5. The binary semi-major axis is plotted against binary mass (filled pentagons) for all the systems containing at least one star with $m \geqslant 5 M_{\odot}$. The periastron separations (open triangles) are also plotted for these systems against the individual component masses, and are joined to their total binary masseand semi-major axis by solid lines. Of note is that some binary systems have only one individual component plotted indicating that the other star has another, closer companion, The dashed line indicates an approximation of the stellar radii and thus periastron separations below this line would force the binary to merge.

2005). Figure $4 \mathrm{~b}$ plots the deduced semi-major axis and periastron separations for the same system as in Figure 4a. From separations of order 100 AU at masses of several solar masses, the binary evolves to a semi-major axis of order 1 AU by the time the system has accreted up to $30 \mathrm{M}_{\odot}$. This once again roughly agrees with an $R_{\text {bin }} \propto M_{\text {bin }}^{-2}$ evolution as expected for accretion from a turbulent medium. Figure $4 \mathrm{~b}$ also shows that the periastron separation decreases more dramatically than does the semi-major axis and attains separations of order the size of high-mass stars $\left(R_{\text {peri }} \lesssim 0.05 \mathrm{AU}\right)$. In fact, at several points, the deduced semi-major axis is smaller than the stellar radii of the stars. Such systems can reasonably be expected to undergo stellar mergers. 


\section{Binary Systems and Stellar Mergers}

The distribution of binary separation as a function of mass is shown for all the highmass binary systems (where at least one star has $m \geqslant 5 M_{\odot}$ ) in Figure 5 . This figure plots the binary's semi-major axis against the total binary mass (filled pentagons) and the individual component masses against the periastron separations (open triangles), linked by solid lines for each system. The more massive stars are generally in binaries with semi-major axes less than 10 AU and periastron separations less than 1 AU. In fact, of the 10 stars at the end of the simulation with masses $m \geqslant 10 M_{\odot}$, all ten are in binary systems $R_{\text {semi }} \leqslant 100$ AU and 8 of them have $R_{\text {semi }} \leqslant 10$ AU.

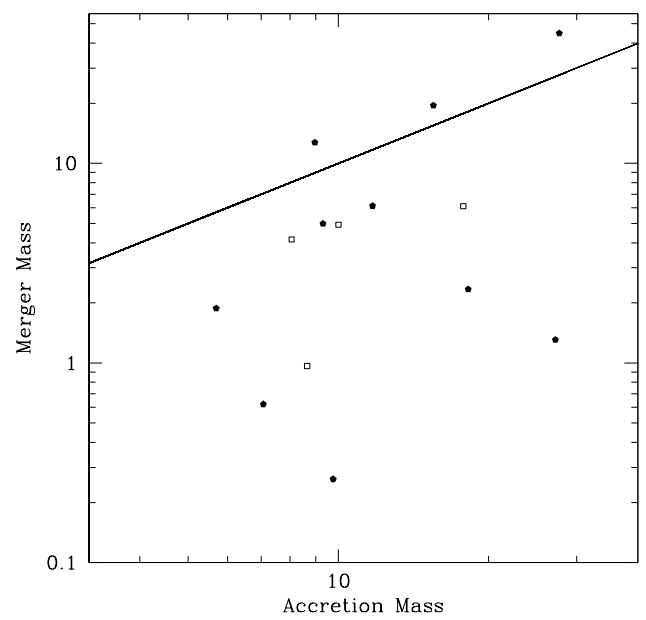

Figure 6. The mass gained in binary mergers, based on having periastron separations smaller than one half of the primary's radius, is plotted against the mass acquired through gas accretion. The final products are plotted as filled pentagons while open squares denote merger products which subsequently merged with a more massive star. The mergers are estimated by calculating the binary properties throughout the simulation and ensuring that each star can only merge once with a more massive star. The solid line denotes equal mass being acquired through mergers and accretion.

Of greater potential importance is that at a given time, a number of the binary systems have periastron separations close to or below the expected stellar radii of high-mass stars. Binary mergers can thus be expected to be a regular occurrence in high-mass star formation. Figure 6 quantifies the expected contribution of binary mergers to the growth of high-mass stars. We see that 3 of the stars are expected to double their mass through mergers and that the most massive star would triple its mass to $\approx 100 M_{\odot}$.

The potential for binary systems to merge and form the most massive stars is an intriguing solution to the difficulty of overcoming the radiation pressure of the forming high-mass star. A binary system with a semi-major axis of a few AU can be easily perturbed by an encounter with a third star that passes near the binary. This encounter radius is significantly larger than the size-scale for direct collisions and thus drastically reduces the requirement of an ultradense cluster. If we consider an eccentric binary of semi-major axis $1 \mathrm{AU}$, the stellar density $n$ of order $10^{6} \mathrm{stars}_{\mathrm{pc}}^{-3}$ is required to have another star pass within $R_{\mathrm{enc}} \approx 2 \mathrm{AU}$, of order the apastron separation, in $t_{\mathrm{enc}} \leqslant 10^{6}$ years. An important implication of this work is that the most massive stars are more 
likely to be single stars, or at least not in a close binary system. This is due to their being merger products and thus the binary system is destroyed in forming this very massive star.

\section{Discs and Winds}

One of the observational signatures of the mode of high-mass star formation has commonly been taken to be the presence of a disc-like structure and collimated outflows. The reasoning is that discs should not exist in the dense cores of clusters where mergers might occur due to the disruption of the passing stars. Unfortunately this is not a clear indication as rotationally supported discs are found to be present in the dense stellar clusters in the simulations of massive star formation (Fig. 7). This occurs due to the continual replenishment from continued infall into the cluster. Thus even though the discs are disturbed by the penetrating encounters of the numerous stars in the environment, it can exist and is rotationally supported over the timescale of the simulation.

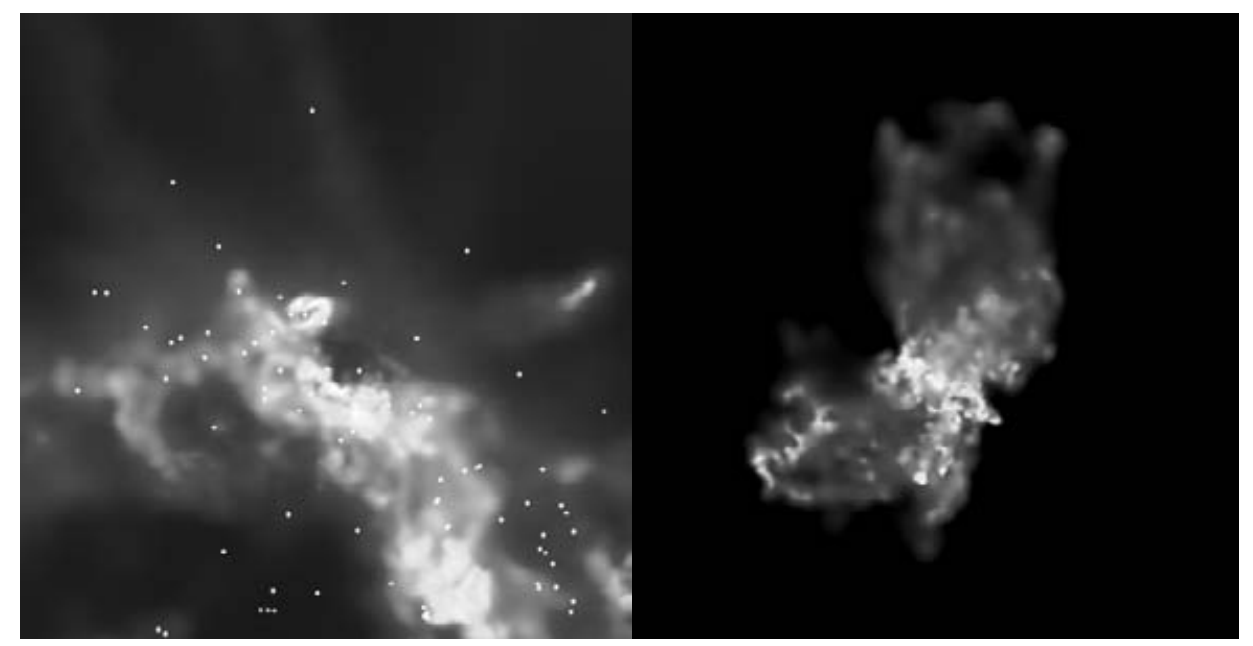

Figure 7. The circumbinary disc that forms around the massive stars in the centre of the cluster is shown nearly edge-on at left while the disc-collimated wind is shown at right from the same viewpoint (image scale few $10^{3} A U$ ). The disc is present even in the high density core of the cluster due to continual replenishment from the infalling gas. Note that the intrinsically wind is collimated by the external distribution of gas in the circumbinary disc.

These structures can even collimate otherwise spherical winds. Using SPH simulations of winds, we have followed the interaction of the wind with the external environment. The disc, and any other non-spherical distribution of circumstellar gas, halts the outflows in its direction such that the emergent outflow is collimated into a bipolar-like shape.

\section{Conclusions}

Numerical simulations show how massive stars can form through accretion and mergers in the cores of stellar clusters. This allows massive star formation to be understood in the context of low-mass star formation and the production of a full initial mass function. The massive stars acquire their mass due to the combined potential of the stellar cluster which funnels the mass down to the core there to be accreted by the proto-massive star. The 
infall of gas into the cluster is accompanied by the infall of newly formed low-mass stars providing a direct link between the formation of massive stars and the formation of the stellar clusters in which they reside. Accretion rates onto the massive stars are of order $10^{-4} M_{\odot} \mathrm{yr}^{-1}, 100$ times larger than that onto the lower-mass stars. These accretion rates are insufficient to overcome the radiation pressure onto dust grains implying an upper limit of stellar masses due to accretion alone in the range 30 to $40 \mathrm{M}_{\odot}$.

Accretion can also explain the high frequency of close binary systems amongst highmass stars. These systems form as wide low-mass binaries and evolve to close higher masses systems as mass is added through accretion. The close binary systems formed in this way can be expected to merge due to dynamical interactions with other stars in the cluster and thus providing a mechanism to explain the higher-mass stars. This mechanism predicts that the most massive stars $\left(m \geqslant 100 M_{\odot}\right)$ are likely to be single if they formed from the merger of a close binary system.

\section{Acknowledgements}

The computations reported here were performed using the U.K. Astrophysical Fluids Facility (UKAFF).

\section{References}

Beech M., Mitalas R., 1994, ApJS, 95, 517

Bally J., Zinnecker H., 2005, AJ, 129, 2281

Bate M. R., Bonnell I. A., Price N. M., 1995, MNRAS, 277, 362.

Bate M. R., Bonnell I. A., Bromm V., 2002, MNRAS, 336, 705

Bate M. R., Bonnell I. A., 1997, MNRAS, 285, 33

Bate M. R., 2000, MNRAS, 314, 33

Bonanos A. Z., Stanek K. Z., 2005, ASPC, 332, 257

Bonnell, I. A., Bate, M. R., 1994, MNRAS, 271, 999

Bonnell, I. A., Bate, M. R., 2002, MNRAS, 336, 659

Bonnell, I. A., Bate, M. R., Clarke, C. J., \& Pringle, J. E., 2001a, MNRAS, 323, 785

Bonnell I. A., Bate M. R., Vine S. G., 2003, MNRAS, 343, 413

Bonnell, I. A., Bate, M. R., \& Zinnecker, H., 1998, MNRAS, 298, 93

Bonnell I. A., Clarke C. J., Bate M. R., Pringle J. E., 2001, MNRAS, 324, 573.

Bonnell I. A., Vine S. G., Bate M. R., 2004, MNRAS, 349, 735

Clarke C.J., Bonnell I. A., Hillenbrand L. A., 2000, , in Protostars and Planets IV (eds V. Mannings, A. P. Boss and S. Russell), 151.

de Wit W.J., Testi L., Palla F., Zinnecker H., 2005, A\&A, in press

Dobbs C.L., Bonnell I.A., Clark P.C., 2005, MNRAS, in press

Edgar R., Clarke C., 2004, MNRAS, 349, 678

García B., Mermilliod J. C., 2001, A\&A, 368, 122

Garmany C. D., Conti P. S., Massey P., 1980, ApJ, 242, 1063

Klessen R. S., Burkert A., 2000, ApJS, 128, 287

Klessen R. S., Burkert A., Bate M. R., 1998, ApJ, 501, L205

Lada C. J., Lada, E. 2003, ARA\&A, 41, 57

Larson, R.B., 2003, Rep. Prog. Physics, 66, 1651

Mason B. D., Gies D. R., Hartkopf W. I., Bagnuolo W. G., Brummelaar T. T., McAlister H. A., 1998, AJ, 115, 821

McKee, C.F., Tan, J.C., 2003, ApJ, 585, 850

Preibisch T., Balega Y., Hofmann K., Weigelt G., Zinnecker H., 1999, NewA, 4, 531

Stahler S. W., Palla F., Ho P. T. P., 2000, prpl.conf, 327

Wolfire M.G., Cassinelli J.P., 1987, ApJ, 319, 850

Yorke H., Krügel E., 1977, A\&A, 54, 183

Yorke H., Sonnhalter, C., 2002, ApJ, 569, 846 